\title{
Surface investigation on prototype cavities for the European X-ray Free Electron Laser
}

\author{
W. Singer, X. Singer, S. Aderhold, A. Ermakov, and K. Twarowski \\ Deutsches Elektronen-Synchrotron DESY, Notkestrasse 85, DE-22603 Hamburg, Germany \\ R. Crooks \\ Black Laboratories, LLC, 116 Villa Road, Newport News, Virginia 12050, USA \\ M. Hoss, F. Schölz, and B. Spaniol \\ W.C. Heraeus, Heraeusstrasse 12-14, DE-63450 Hanau, Germany \\ (Received 15 November 2010; published 6 May 2011)
}

\begin{abstract}
The accelerating gradient Eacc of X-ray Free Electron Laser (XFEL) prototype cavities manufactured at the industry and treated at DESY demonstrates wide-range scattering from 15 to $41 \mathrm{MV} / \mathrm{m}$. Most cavities satisfy the XFEL specification. Few cavities with low performance $(15-17 \mathrm{MV} / \mathrm{m})$ are limited by quench without field emission. The T-map analysis detected quench areas mainly close to the equator. Optical control by a high resolution camera has been applied and allowed to monitor the defects in some cases with good correlation to T-map observation. In order to understand the cause of reduced performance and get more detailed information of the origin of defects, some samples have been extracted from two cavities and investigated by light microscope, digital light microscope with 3D profile measurement, scanning electron microscope SEM, energy dispersive x-ray analysis, and Auger spectroscopy. The electron backscattered diffraction method in a SEM is used to make localized measurements of the lattice curvature. Several surface flaws with sizes from a few $\mu \mathrm{m}$ to hundreds of $\mu \mathrm{m}$ detected by microscopy. The defects can be separated into two categories. The first category of defects consists of foreign elements (often an increased content of carbon). Inclusions with increased content of carbon adhere on the surface and presumably have a hydrocarbon nature. Deviation from a smooth surface profile characterizes the second type of defects (holes, bumps, and pits). Some holes and bumps were found directly in the welding seam. The hot spots in the heat-affected zone (HAZ) of the equator welds have been partially associated with pits too. The study correlates the location of pits with the presence of plastic strain found to remain after welding. Pits near the HAZ were found either coincident with or near areas of high strain. Pits away from the weld were often found at grain boundary triple junctions.
\end{abstract}

DOI: 10.1103/PhysRevSTAB.14.050702

PACS numbers: 41.60.Cr, 52.59.Rz

\section{INTRODUCTION}

As described in [1], approximately 50 prototype cavities have been mechanically manufactured at the industry by two vendors, treated (partially at the factory and partially at DESY) and rf-tested at DESY. The treatment for all cavities was removal by electropolishing (EP) of a $110-140 \mu \mathrm{m}$ surface layer, followed by an ethanol rinse and $800^{\circ} \mathrm{C}$ annealing under UHV conditions. Two options of final treatment have been applied. Final EP of 40-50 $\mu \mathrm{m}$ with subsequent ethanol rinse, ultrapure high pressure water rinsing (HPR) and $120^{\circ} \mathrm{C}$ bake or alternatively a final buffered chemical polishing (BCP) of $10 \mu \mathrm{m}$ (called Flash BCP), HPR and $120^{\circ} \mathrm{C}$ bake. The XFEL

Published by the American Physical Society under the terms of the Creative Commons Attribution 3.0 License. Further distribution of this work must maintain attribution to the author(s) and the published article's title, journal citation, and DOI. requirements are fulfilled with a yield of approximately $90 \%$.

The main task of this work was concentrated on five cavities (Z110-Z111, Z130-Z132), produced by one vendor, that have an Eacc below $20 \mathrm{MV} / \mathrm{m}$. As can be seen in Fig. 1(a), the Qo(Eacc) behavior of these cavities seems to be similar: rather small change of Qo between $5-15 \mathrm{MV} / \mathrm{m}$ and after that a quench at $15-17 \mathrm{MV} / \mathrm{m}$. Four cavities were treated by final EP and only one cavity (Z111) had the flash BCP final treatment. One of the cavities (Z110) was subjected to the attempt to improve performance by post-purification with titanium by annealing at $1400^{\circ} \mathrm{C}$ for 4 hours and subsequent $\mathrm{BCP}$ of approximately $100 \mu \mathrm{m}$. Unfortunately, this did not lead to an improvement of the accelerating gradient.

In the past 15 years of cavity production for TESLA Test Facility (FLASH), a case of failure by the fabrication resulted in a sequence of six cavities (S7-S12) with reduced performance [Fig. 1(b)]. Thorough analysis at that time together with the company allowed one to find out that after local grinding the surface was not sufficiently cleaned 

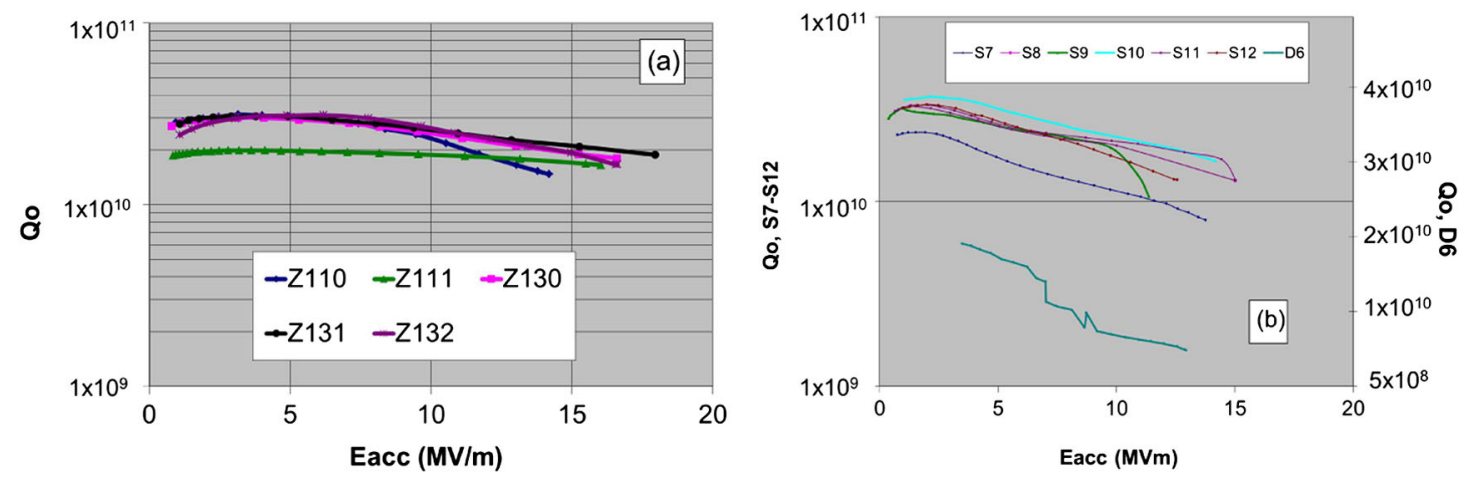

FIG. 1. (a) Cavities of XFEL prototype manufactured with Eacc below $20 \mathrm{MV} / \mathrm{m}$; (b) cavities S7-S12 and D6 of the 1st generation of FLASH fabrication.

and contamination probably penetrated into material during electron beam welding.

It is interesting that two series of cavities with reduced performance demonstrate comparable behavior [Figs. 1(a) and 1(b)]. The contaminated cavities S7-S12 exhibit a bit more steep $Q$ reduction. In both cases Eacc is limited by a strong quench below $20 \mathrm{MV} / \mathrm{m}$.

Analysis of the manufacturing steps of $Z$ cavities at the company did not contribute to an understanding of the reason for failures. It was decided to cut the cavities and investigate the surface in the hot-spot areas that have been indicated by the DESY T-map system for nine-cell cavities. Cavity destruction for investigation is a rare action. Only one of those cavities intended for FLASH (D6) with affected performance [see Fig. 1(b)] was destroyed in the past [2]. The analysis of the obtained samples was successful. A material flaw was detected clearly at the location identified by the T-map. A weak superconducting local defect cluster of the area of approximately $0.5 \mathrm{~mm}^{2}$ with increased tantalum content was responsible for the local heating, that caused the quench and specific $\mathrm{Q}($ Eacc) behavior [Fig. 1(b)], in particular, the abrupt Qo drop at ca. $7 \mathrm{MV} / \mathrm{m}(Q$ switch $)$.

Several samples were taken from cavities Z111 (BCPflash treated) and Z130 (final EP treated). The separation of the samples from cavity was done using milling machine and special adapter allowing separating samples of disklike shape with a diameter of ca. $35 \mathrm{~mm}$ (Fig. 2). The rotation speed of the milling cutter did not exceed $50 \mathrm{rpm}$. The damage of the sample's surface by metal filings or by the sample falling down inside the cavity was avoided. The cutting was done in a conventional environment without any lubrication. The temperature of the samples during separation did not exceed $50^{\circ} \mathrm{C}$. Cavities additionally were previously high pressure water rinsed. The samples were taken from cavity quench areas or areas with increased heating (hot spots) that were localized by T-mapping during vertical $\mathrm{rf}$ test.

Light microscopy, 3D microscopy, SEM including electron backscattered diffraction (EBSD), energy dispersive $\mathrm{x}$-ray analysis (EDX), and Auger spectroscopy were used

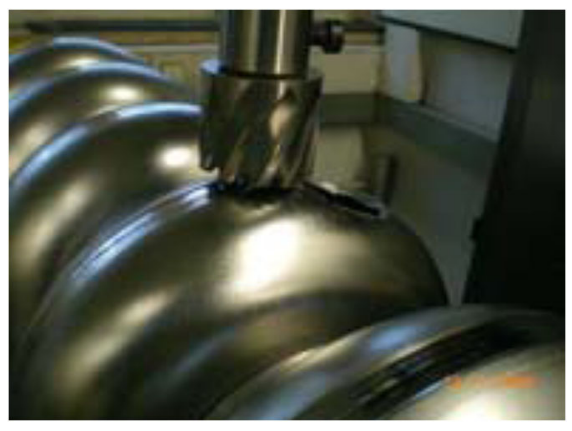

FIG. 2. Separation of samples for surface investigation

for investigation. Several surface flaws were detected. It has to be mentioned that the suspicious area localized by T-mapping has a dimension of square centimeters, while the size of suspicious flaws lays in the $\mu \mathrm{m}$ order of magnitude. Therefore the absolutely unambiguous conclusion about which of the detected defects is responsible for the main heating in the local area is currently not possible. The detected defects can be separated in two categories. The first category of defects indicates foreign elements. Deviation from a smooth surface profile characterizes the second type of defects (holes, bumps, and pits).

\section{DEFECTS WITH FOREIGN MATERIAL}

Foreign material inclusions in the hot-spot areas were detected in both cavities.

Some examples of the foreign material inclusions can be seen below. Many surface inclusions with sizes from a few $\mu \mathrm{m}$ to hundreds of $\mu \mathrm{m}$ were detected by SEM on two samples separated from cell 1 of the cavity Z130 [Figs. 3(a)-3(c)]. One inclusion indicates increased content of iron [Figs. 4(a) and 4(b)].

Most inclusions indicate increased content of carbon. Some inclusions indicate increased content of oxygen as well as carbon [see, for example, Fig. 5(a) and 5(b)].

Similar black spots of different shapes have been observed in the cavity Z111 too [see, for example, Fig. 6(a)]. At first glance it seems that the black spots lie on the 

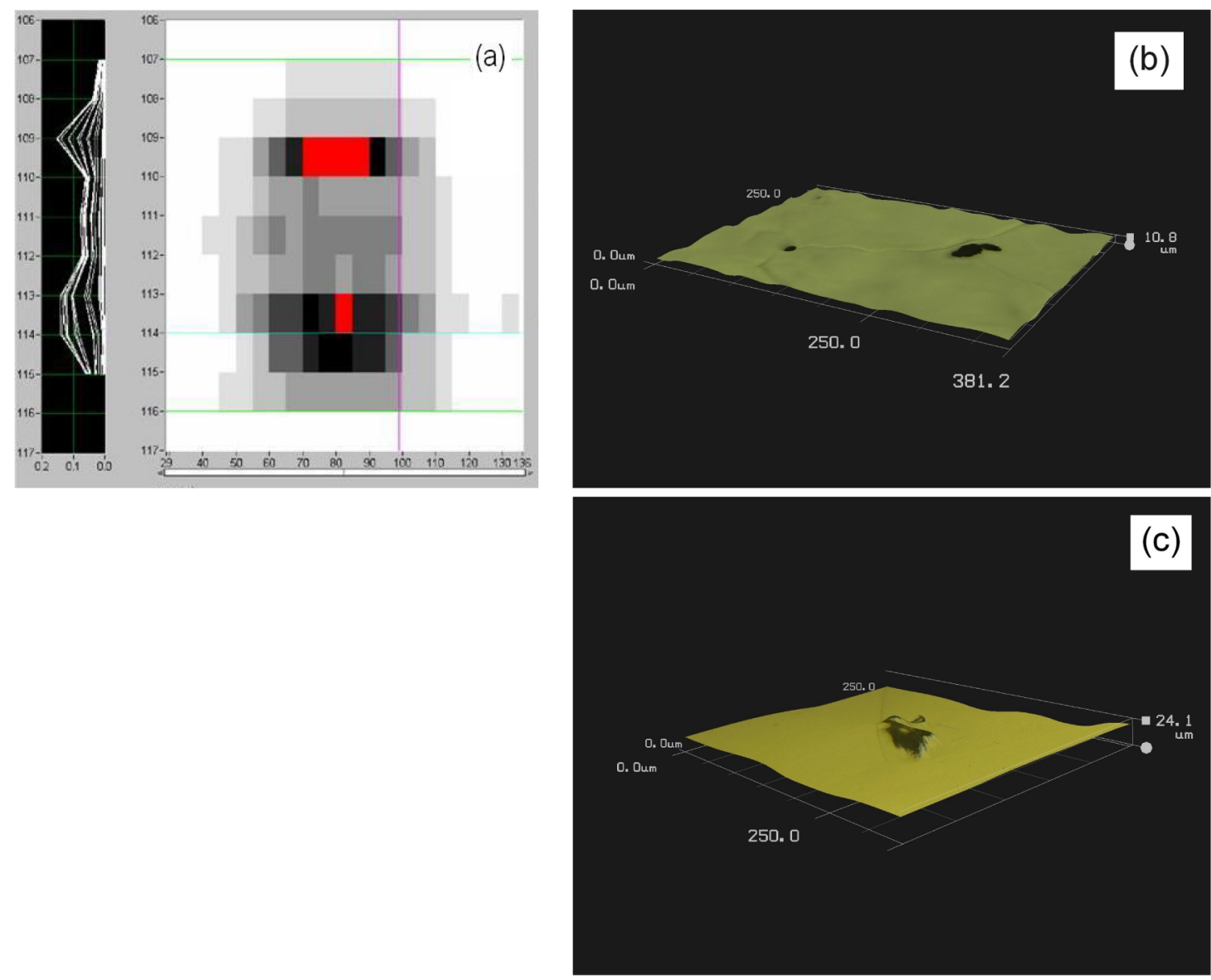

FIG. 3. (a) T-map for $\pi$ mode with hot spots in the equator area of cavity Z130 and away from the equator caused quench at $16 \mathrm{MV} / m$; (b), (c) images of defects detected in the hot-spot areas shown in (a).
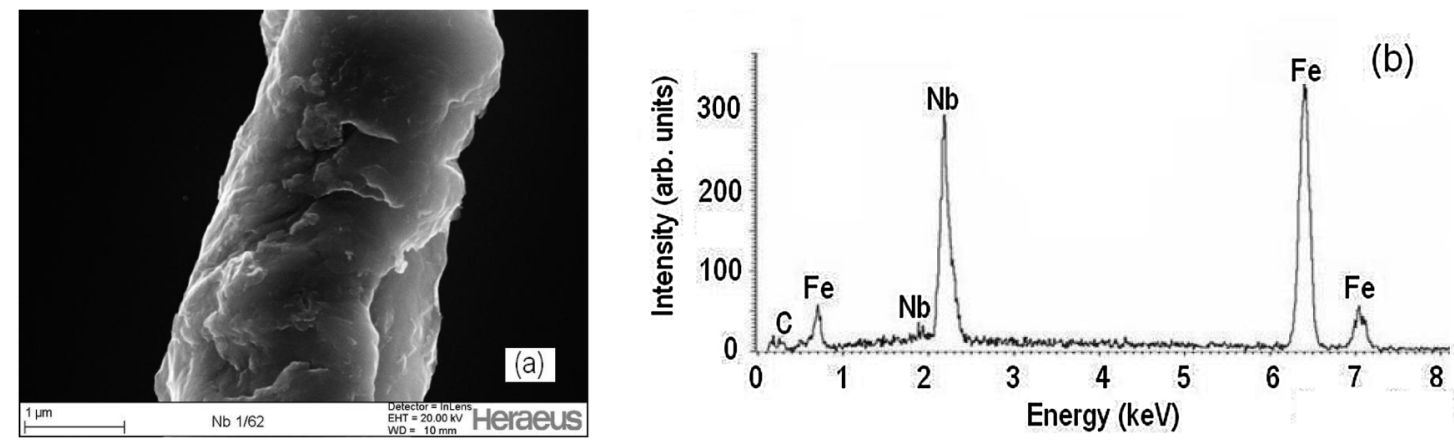

FIG. 4. (a) SEM image of the foreign material inclusion; (b) EDX element analysis of the image of (a).

surface [Fig. 3(b) upper image, for example] and do not stick in, but more precise analysis shows that the material of the black spots adheres to the surface [Fig. 3(c) beneath].

One of the black areas [see image in the Fig. 6(a)] with pronounced carbon signal in the Auger spectrum [Fig. 6(b)] was removed by ion sputtering. The black spot and the carbon signal disappeared only after many cycles of sputtering and removal of a layer of $500 \mathrm{~nm}$ [Figs. 7(a) and 7(b)].

\section{TOPOGRAPHICAL DEFECTS IN THE EQUATOR WELD}

There is a lot of discussion in the community, whether or not and, if yes, how much the surface topography influences the cavity performance. At least two found topographical defects are doubtless correlated with cell performance. In both cases the defects were in the equator weld area.

Figure 8(a) shows the T-map for (3/9) $\pi$ mode excitation. Quench at $23,3 \mathrm{MV} / \mathrm{m}$ was found in cell 5 of 

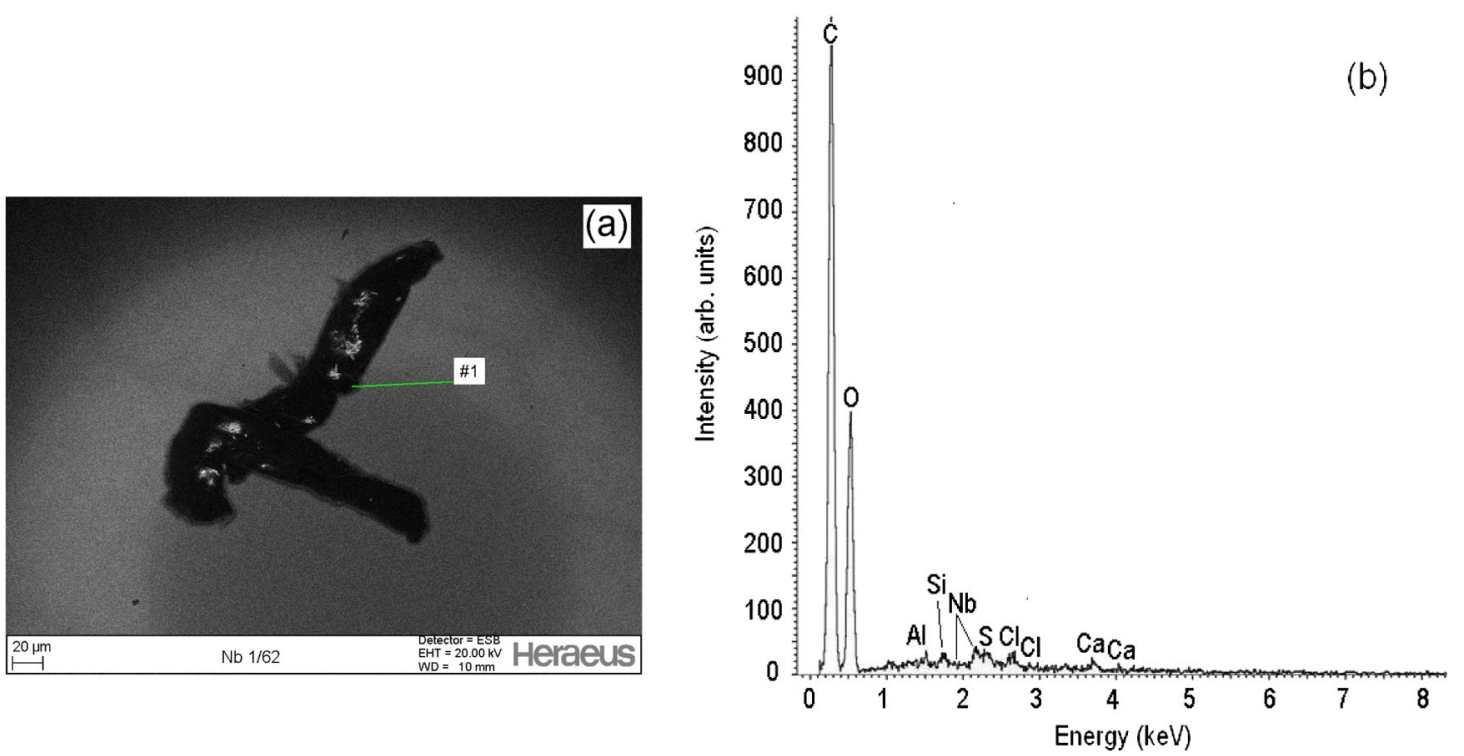

FIG. 5. (a) SEM image of the black spot represented in Fig. 3(b); (b) EDX element analysis of the black spot represented by (a).
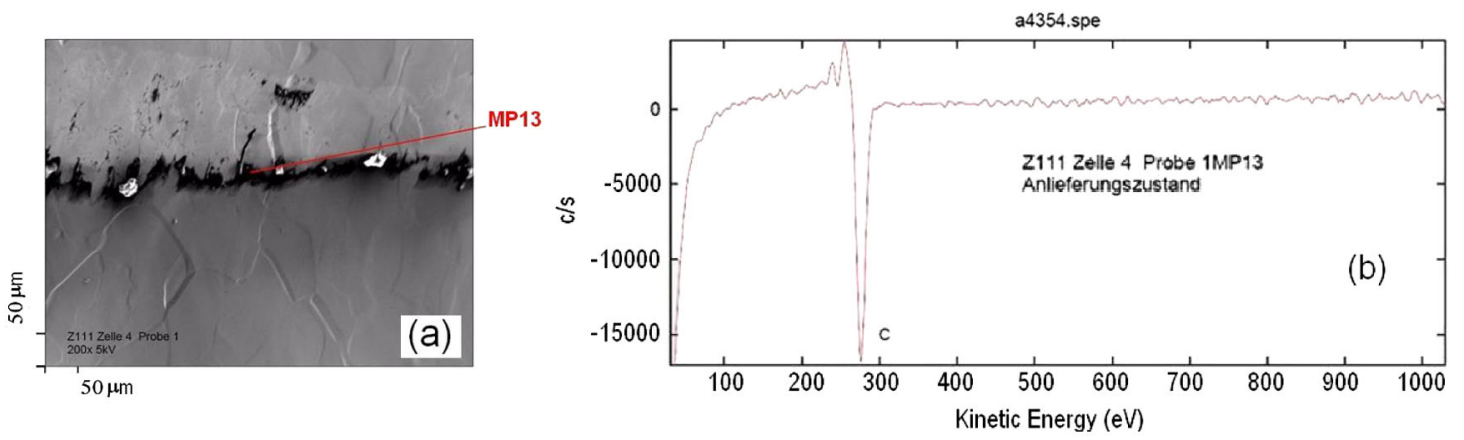

FIG. 6. (a) Area of black spots detected in the cavity Z111; (b) Auger spectrum of the black spots of (a) indicating the presence of carbon.
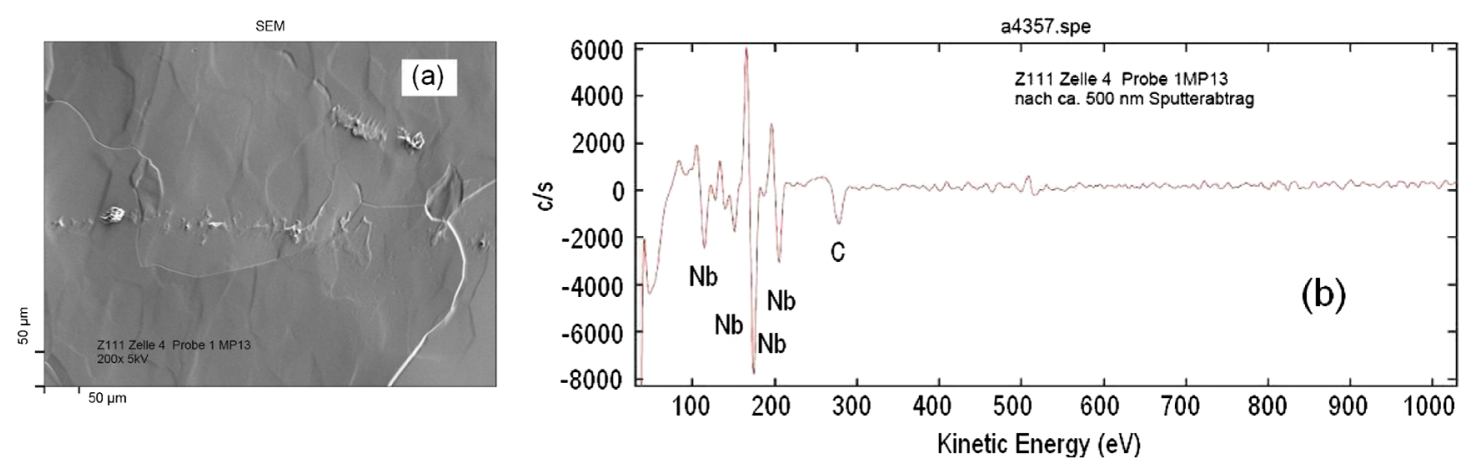

FIG. 7. (a) Area shown in Fig. 6(a), but after ion sputtering and removal of the layer of $500 \mathrm{~nm}$; (b) Auger spectrum of the area shown in (a) (conventional spectrum of pure niobium).

the cavity Z130. Optical inspection done on the cavity by the high resolution camera in use at DESY discovered a topographical defect of $1 \mathrm{~mm}$ size on the interface between the welding seam and heat-affected zone [Fig. 8(b)]. The defect area was studied more carefully on the separated sample. No foreign material was monitored. The topography of the defect with a big distance between the peak and the valley (more than $150 \mu \mathrm{m}$ ) can be seen on images of light and 3D microscopes [Figs. 9(a) and 9(b)]. 
Another topographical defect that limited the accelerating gradient by quench at $16 \mathrm{MV} / \mathrm{m}$ was detected in cell 6 of cavity Z111 on the equator weld. A group of beads of $1.5 \mathrm{~mm}$ width were observed in the heating location [Fig. 10(a)] by optical inspection [Fig. 10(b)].

More details have been observed on the samples, separated from this area: several holes positioned mainly
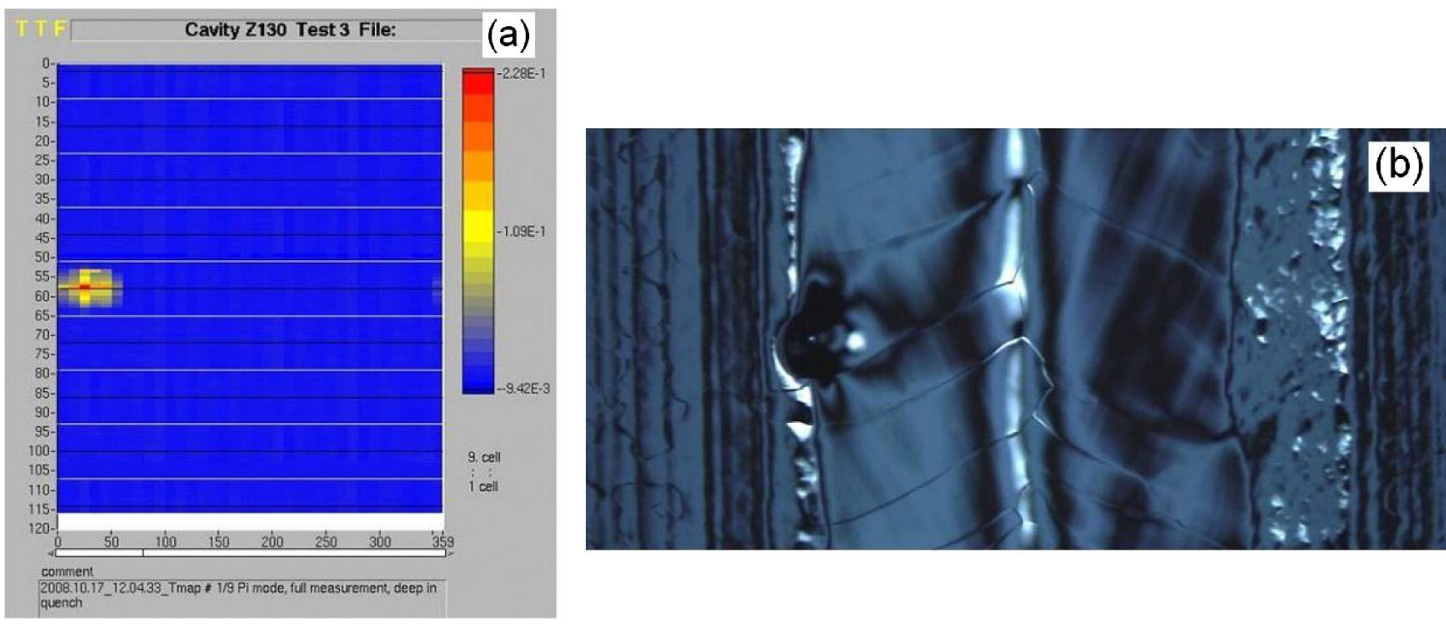

FIG. 8. (a) T-map for (3/9) $\pi$ mode excitation of the cavity Z130; (b) optical inspection image of the hot spot represented in (a).
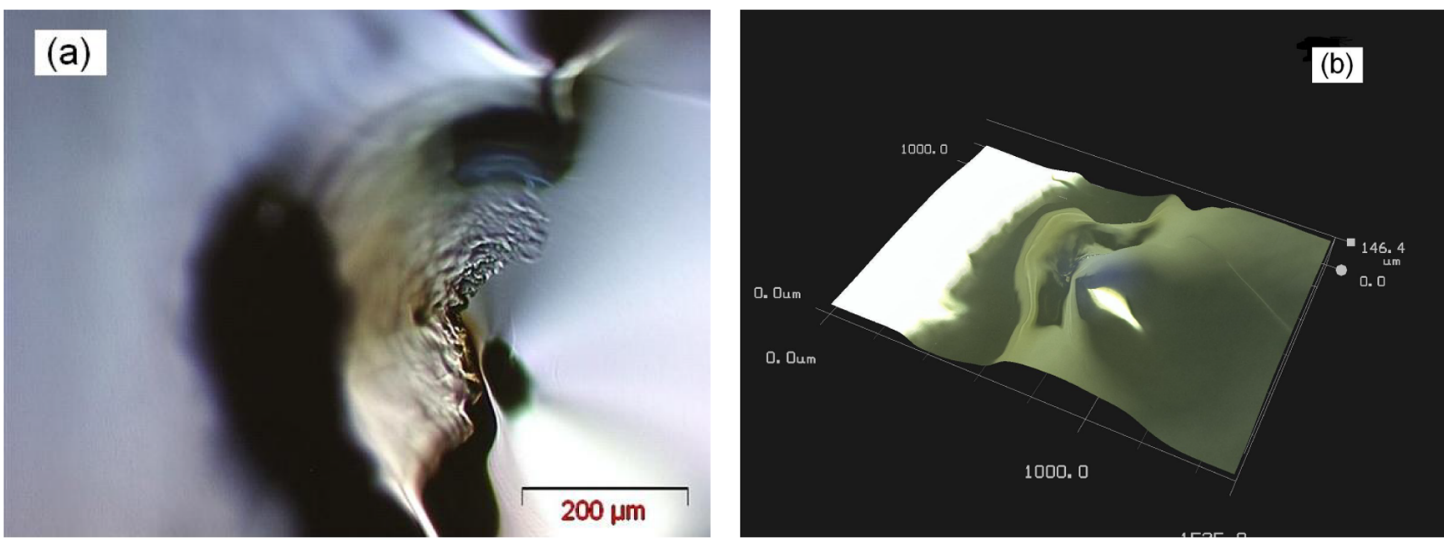

FIG. 9. (a) Light microscope image of the hot spot represented in Fig. 8(a); (b) 3D microscope image of the hot spot represented in Fig. 8(a).
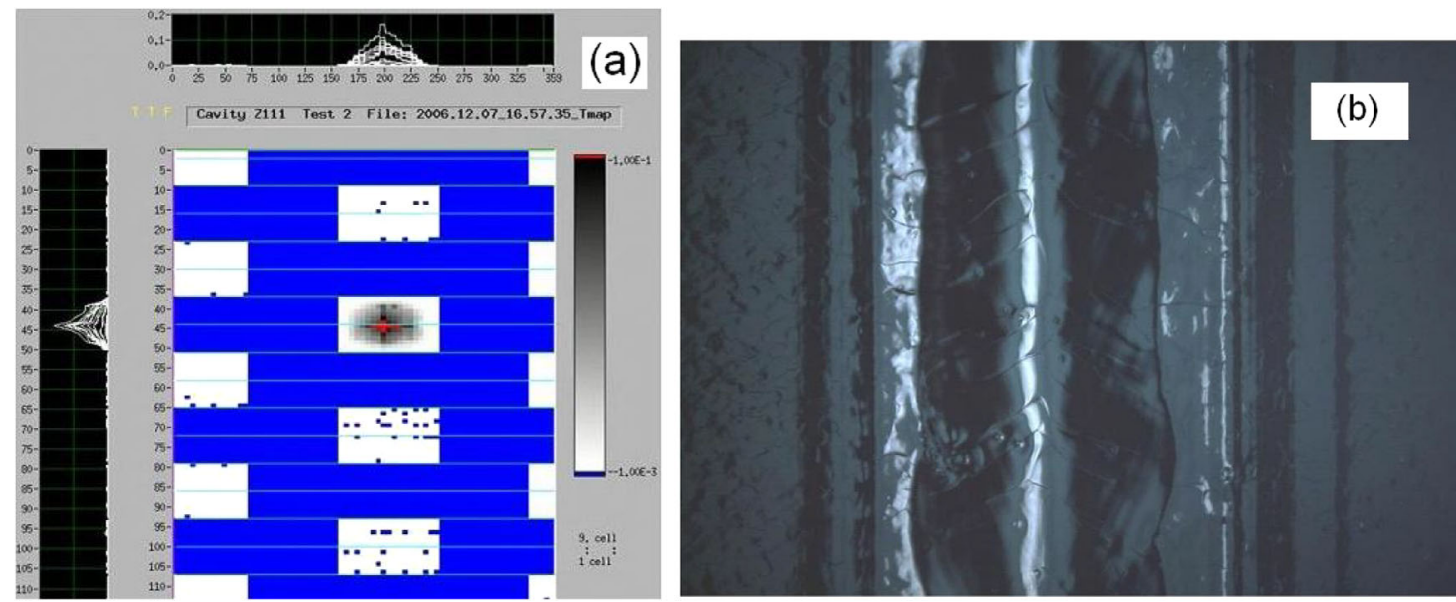

FIG. 10. (a) T-map of cavity Z111; (b) high resolution camera inspection of the hot spot of (a). 

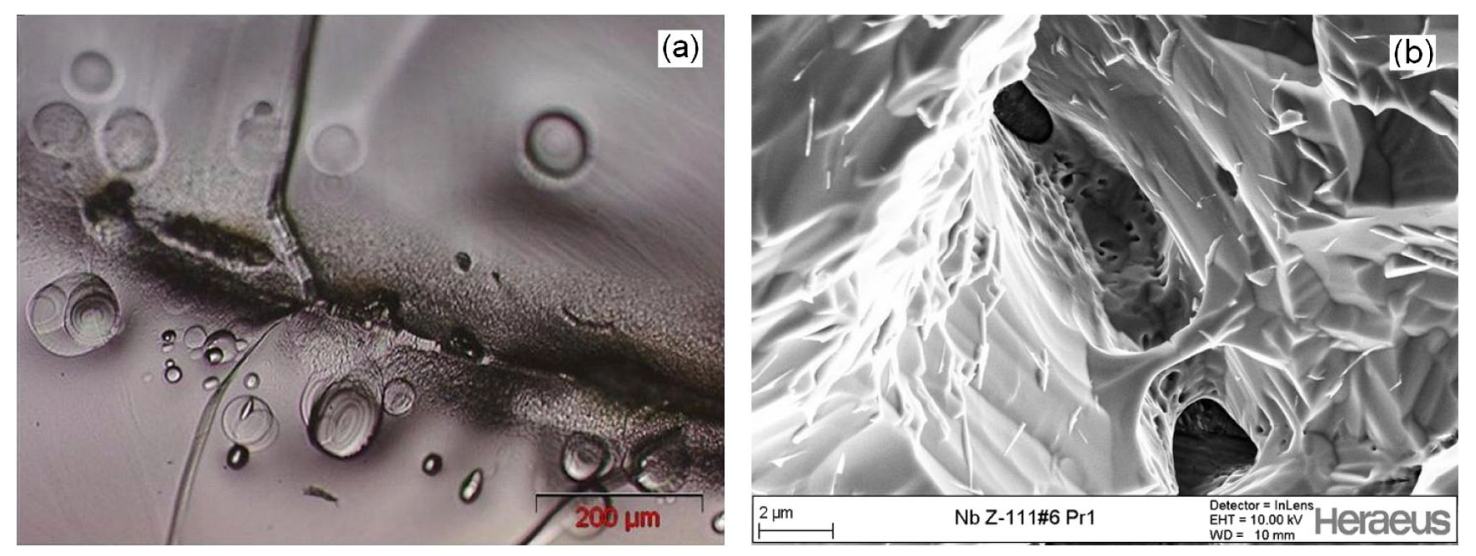

FIG. 11. (a) Light microscope image of the hot spot represented in Fig. 10(a); (b) magnified SEM image of holes represented in (a).

along a grain boundary were clearly visible in the light microscope image [Fig. 11(a)]. Smaller holes inside of the bigger hole were detected by high SEM resolution [Fig. 11(b)]. The impression gained was that of local corrosion taking place in this area. Several etching pits were positioned around corrosion holes.

\section{TOPOGRAPHICAL DEFECTS OUTSIDE THE EQUATOR WELD}

The investigation of the influence of work hardening, welding, EP conditions, etc., on the pitting creation and its role in the heating load of the cavities is currently a very actual topic in the SRF community (see, for example, [3-6]). A lot of experiments are carried out on coupons (see, for example, $[5,6]$ ). Unfortunately the fabrication and treatment conditions of nine-cell elliptical cavities cannot be completely simulated with coupons. Therefore the seldom opportunity to observe and analyze the pitting in a treated real nine-cell cavity was very exciting.

A lot of etching pits of different shapes and sizes were observed on the inside surface of both cavities, especially on Z111.

Several samples of BCP flash treated Z111 cavity interior surfaces with suspicion for the hot spots were studied in a digital microscope with 3D profile measurement and SEM, equipped with a TSL Hikari electron backscattered diffraction (EBSD) system.

The curved samples were tilted to approximately $70^{\circ}$ to horizontal. Data were collected in a hexagonal grid pattern with a point-to-point distance of 0.5 to $2 \mu \mathrm{m}$. The crystal orientations were calculated from the pattern images through a Hough transformation method using TSL OIM software. Data plotted included a map comprised of pixels showing the pattern contrast, which is shown as a grey scale image (image quality map). The crystallographic data were plotted as inverse pole figure maps (IPF) or grain reference orientation deviation maps (GROD). Inverse pole figures were used to show the orientation of the crystal axis normal to the surface, and give a view of the grain size and a qualitative indication of the amount of distortion of the crystal lattice within each grain. High angle grain boundaries (with pixel to pixel changes of $15^{\circ}$ ) are shown as black lines. The grain reference orientation deviation is a quantitative display of the deviation of points within each grain from the point of minimum lattice distortion. The minimum is essentially the flattest point of the crystal lattice within each grain. A quadrapole backscattered detector was used in conjunction with measurement software on a Hitachi SEM to determine the pit morphology.

Topography of the pitting as well as the influence of the crystallographic grain orientation and cold-work residues on the pitting is analyzed. A few images were taken with a backscattered imaging system designed to measure the three-dimensional morphology of features. Many pits were studied which are not included in this paper. The results below are representative.

Two categories of samples were examined from different regions of the cavity: pits near the heat-affected zone HAZ of the equator weld and pits further down the interior cavity wall. It seems that the nature of the pits in the HAZ of the welding seams and in the other cell areas are different.

\section{A. Pits near the HAZ}

Figure 12 shows the results from a pit near the HAZ of an equator weld. The pit encloses a region of high strain (high dislocation density). This is evident in the color change of the IPF map and in the GROD map. The GROD map indicates a lattice rotation rate of $3^{\circ} / \mu \mathrm{m}$ within the pit in the absence of any grain boundaries. The pit was within a grain with a surface near the (111) plane.

\section{B. Pits far from the HAZ}

It is interesting to note that the pits far away from HAZ have been detected mostly on the lower half cell, when orienting the vertically positioned cavity with the main coupler at the bottom. 

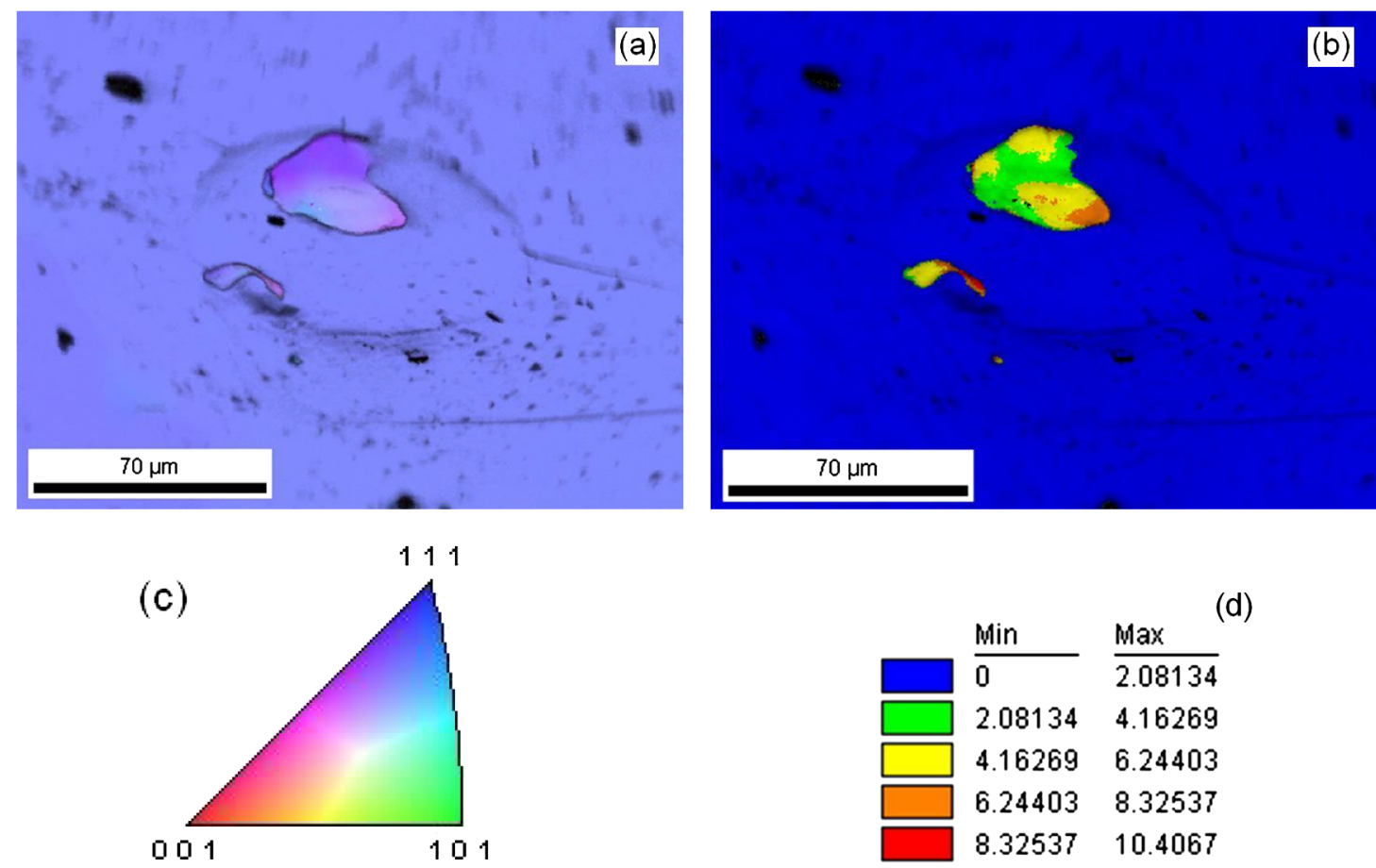

FIG. 12. SEM and EBSD results from pit near the HAZ, sample \#2. $(200 \times 200 \mu \mathrm{m}, 0.5 \mu \mathrm{m}$ resolution): (a) inverse pole figure (pit near [111]); (b) GROD map; (c) key for (a); d) key for (b). Maximum lattice rotation rate within pit of $3^{\circ} / \mu \mathrm{m}$.

The pit shown on Fig. 12 was not near high strain areas but from a grain surface near (111). Another sample shows a grain surface near (001). A larger area scan was done in an attempt to locate any high strain areas near the pit. The pit was on a near (001) grain surface without any nearby strain (Fig. 13).

\section{B. Pit morphology}

The results describing the pit morphology are shown in Figs. 14(a)-14(c). The examined pits were shallow (the depth of the pits lay in the region of 5-20 $\mu \mathrm{m}$ ) and had a flat bottom. The pit walls were mostly tapered.
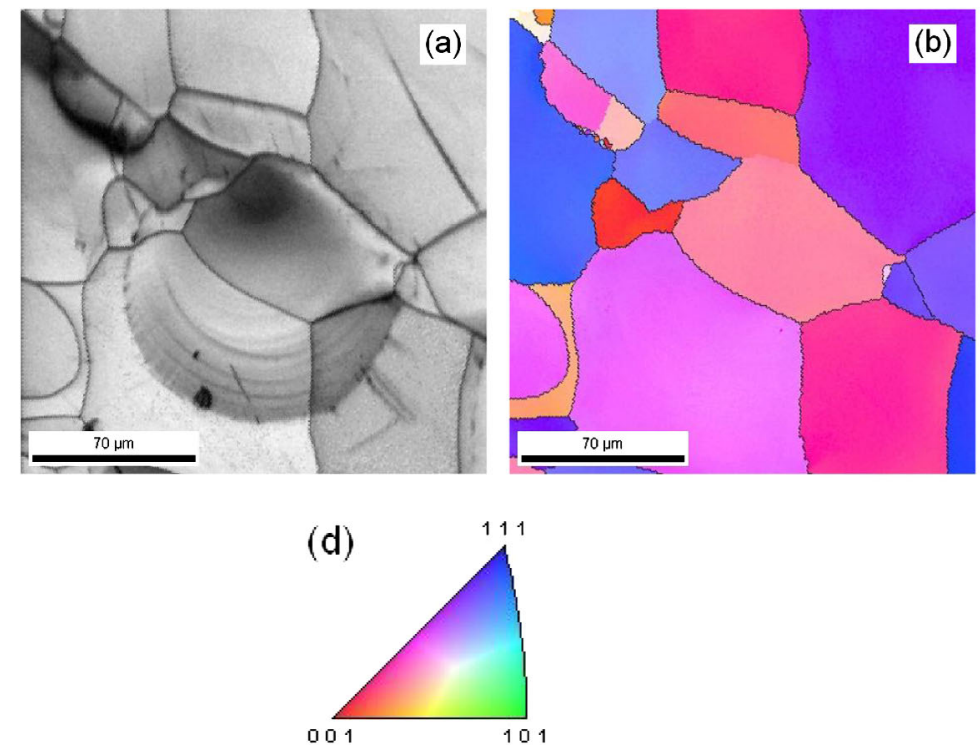

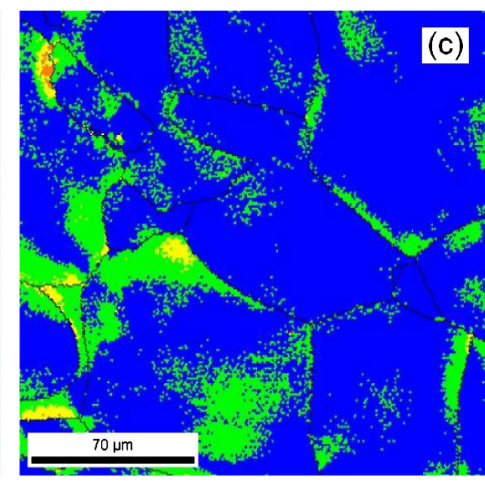

(e)

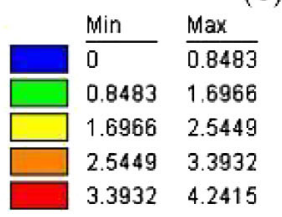

FIG. 13. SEM and EBSD results from pit far from the HAZ, sample \#4. (200 $\times 200 \mu \mathrm{m}, 1 \mu \mathrm{m}$ resolution): (a) image quality map; (b) inverse pole figure (pit near [001]); (c) GROD map; (d) key for (b); (e) key for (c). Maximum lattice rotation rate within pit of $\sim 0.2^{\circ} / \mu \mathrm{m}$. Note "beach" markings. 

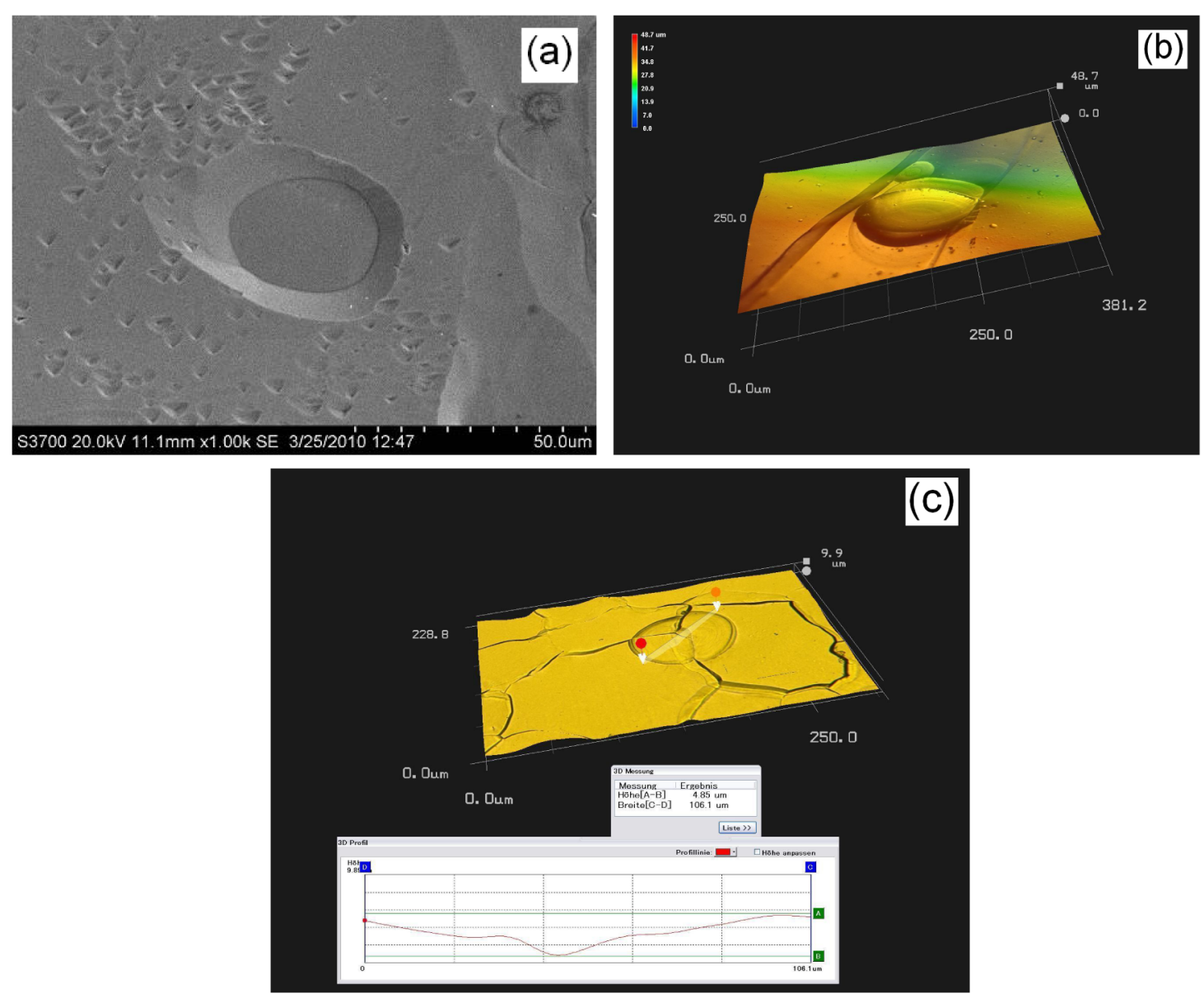

FIG. 14. Examples of images and depth profiles of pits. Pits are shallow and flat.

\section{SUMMARY AND DISCUSSION}

Both foreign material inclusions as well as topographical flaws were found and can be classified as sources of the quench in investigated cavities. Even if the reason for the quenches is understood, the explanation of the reason that such defects appear on the cavity surface is more difficult. Only more or less plausible speculations are possible here.

For example, it could be highly probably supposed that embedding of iron particles happened during deep drawing or handling of subassembly parts at the factory. Particles were already present during EP treatment; the surface of the particles is smooth as usual after EP. On the other hand, they were not present in the niobium sheet. The sheets were provided to the vendor after eddy current scanning [2]. Iron, as a magnetic material, contributed significantly to eddy current signal and definitely would be detected.

The topological defect presented in Figs. 8 and 9 seemingly could be caused at the factory presumably as a result of not sufficient removal of the flake after machining; or imprecise clamping, or possibly a welding error in this location.
More difficult to explain is the cause of the black spots as shown in Figs. 3 and 5-7. The Auger spectrums indicate attendants of hydrocarbons. On one hand, a lot of hydrocarbons surround the electron beam welding (EBW) procedure (not sufficient part degreasing, oil fractions from the diffusion pump, rest of oils on the wall of the EBW chamber, etc.). At the same time it is difficult to imagine, even if the surface were contaminated, how the contaminants could survive after the subsequent treatment including removal of the surface layer of $150 \mu \mathrm{m}$ thick. On the other hand, a lot of hydrocarbon-based material is used into treatment procedure too (polymers, alcohols, etc.) which (if remains on the surface) can adhere during $800^{\circ} \mathrm{C}$ annealing. Final EP removes these remainders possibly less efficiently compared to BCP flash. Suspicion of this could perhaps explain why the bad cavities were mainly EP treated in the end. From this point of view, the corrosion holes in Z111 can be considered as the residues remaining after etching away of the black spots by BCP.

Pits from different regions of the cavity interior surface were compared for orientation of the grain face plane, for lattice curvature within the pit, and for lattice curvature nearby the pit. Pits near the equator weld HAZ tended to be located near regions of high lattice curvature rate, 


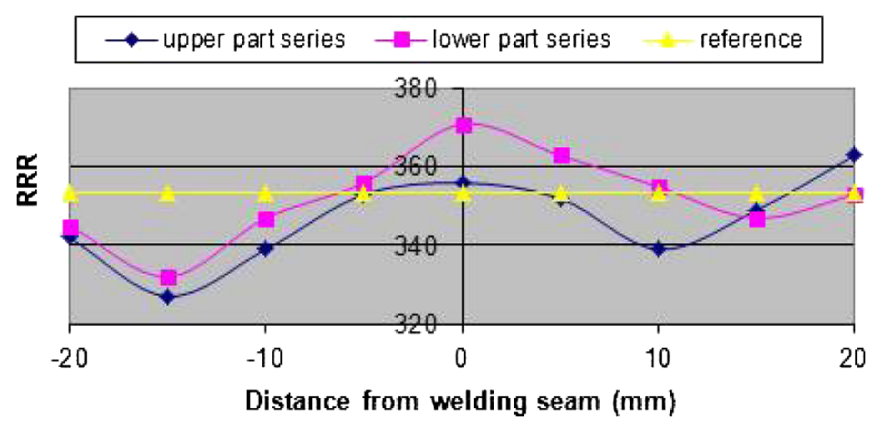

FIG. 15. RRR in welding area (electron beam welding at pressure $2.3 \times 10^{-8} \mathrm{mbar}$ ).

indicative of high stored strain energy. They were found on (111) surface planes, within individual grain.

Pits far from the HAZ tended to occur on grain boundary triple junctions. They were found on both (111) and (001) grain surfaces. Although these pits appeared to be of the same geometry as the HAZ pits, they included peripheral "beach markings" near the edge of the pits. The "beach markings" were not observed on the HAZ pits. The "beach markings" suggest a progressive chemical reaction associated with the pit formation, or a process which occurs during evaporation of a liquid solution.

The HAZ pit regions were cut from areas associated with hot spots during SRF testing. It is plausible that the remnant high stored strain in these regions may be associated with increase interstitial impurities, since interstitial solubility increases in the presence of dislocations due to the low energy interstitial sites which give rise to dislocation atmospheres. The high dislocation density remaining after welding, at the edge of the HAZ, may contribute to a higher interstitial impurity region. In this case the reduction of residual resistivity ratio (RRR) has to be expected. This was indeed observed by authors on the samples electron beam welded at DESY (Fig. 15). Increased content of interstitial impurities (oxygen and nitrogen) in the HAZ was apprized in work [7]. In [8] is demonstrated that the hot-spot precursor forms in the weld HAZ. The images in this work of pits near the HAZ show that high dislocation regions may occur as isolated and small volumes, which would be consistent with the occurrence of hot spots during SRF testing.

There is no apparent connection between the pits far from the HAZ and the stored strain energy. Furthermore, no good correlation between pits and grain orientation has been detected.

The pits in the studied samples from BCP flash treated cavity were shallow and flat. It should be noted that this is quite different from the measurements of pits of electropolished cavities reported in [9]. However, the effect of electropolishing on pit morphology was not examined in the current study.

The action of cavity destruction allowed us to get new information and some new knowledge. At the same time many questions remain: for example, why post purification followed by BCP did not improve the performance of the cavity Z110.

\section{ACKNOWLEDGMENTS}

We would like to thank A. Matheisen and B. van der Horst for the support of the work.

[1] W. Singer et al., "Preparation Phase for $1.3 \mathrm{GHz}$ Cavity Production of the European XFEL," in Proceedings of the First International Particle Accelerator Conference, Kyoto, Japan (2010), THOARA02, http://www .JACoW.org.

[2] W. Singer et al., Part. Accel. 60, 83 (1998).

[3] K. Wanatabe, in Proceedings of the 14th Workshop on RF Superconductivity, Berlin, 2009, pp. 123-128, http:// www.JACoW.org.

[4] X. Zhao, G. Ciovati, C.E. Reece, and A.T. Wu, in Proceedings of the 23rd Particle Accelerator Conference, Vancouver, Canada, 2009 (IEEE, Piscataway, NJ, 2009), http://trshare.triumf.ca/ pac09proc/Proceedings/papers/ we5pfp062.pdf.

[5] L. D. Cooley, D. Burk, C. Cooper, N. Dhanaraj, M. Foley, D. Ford, K Gould, D. Hicks, R. Novitski, A. Romanenko, R. Schuessler, C. Thompson, and G. Wu, Impact of Forming, Welding, and Electropolishing on Pitting and the Surface Finish of SRF Cavity Niobium. Applied Superconductivity Conference, Washington, D.C. (2010), presentation $5 \mathrm{MZ}-01$.

[6] P. M. Michelato and L. Monaco, in Proceedings of the 14th Workshop on RF Superconductivity, Berlin, 2009 (Ref. [3]), THPPO091.

[7] S. Anakhov, X. Singer, W. Singer, and H. M. Wen, in Proceedings of the Second International Symposium on Hydrogen in Matter (ISOHIM), AIP Conf. Proc. No. 837 (AIP, New York, 2006), p. 837

[8] M. Champion et al., IEEE Trans. Appl. Supercond. 19, 1384 (2009).

[9] G. Wu, M. Ge, P. Kneisel, K. Zhao, L. Cooley, J. Ozelis, D. Sergatskov, and C. Cooper, Investigations of Surface Quality and SRF Cavity Performance, Applied Superconductivity Conference, Washington, D.C., 2010, presentation 2LP1C-02. 